\title{
(1) \\ Fundeni \\ Clinical challenges in diagnosis and management of Acute Megakaryocytic Leukemia associated with a Mediastinal Nonseminomatous Germ Cell Tumor \\ Grigoras $O_{2}$, Stancioaica $C_{2}$, Dobrea C 1,2 , Vasilache D 1,2 Parascan L 1 Serban C 2 , Popa C 1,2, Gheorghe A. 2 , Coriu D 1,2
}

${ }_{1}$ Department of Hematology-Fundeni Clinical Institute, University of Medicine and Pharmacy "Carol Davila", Bucharest, Romania; ${ }^{2}$ Center of Hematology and Bone Marrow Transplantation, Fundeni Clinical Institute, Bucharest, Romania.

\section{BACKGROUND}

The connection between a mediastinal nonseminomatous germ cell tumor and an Acute Megakaryocytic Leukemia (AML-M7) is highly uncommon and has a poor prognosis. There seems to be a predisposition for this twofold malignancy in young adults males.

\section{CASE REPORT}

Here we report a male patient (26 years) admitted in our department for chest pain, fever, and tickly cough, severe pancytopenia $(\mathrm{WBC}=7.330 / \mathrm{mmc}, \mathrm{Hb}=$ $10.6 \mathrm{~g} / \mathrm{dl}, \quad P L T=10.000 / \mathrm{mmc}$ ) and elevated serum levels of LDH (4760U/L), AFP (over 1000ng/ml), $\beta$-HCG (337 IU/L). The CT-scan revealed an extensive anterior mediastinal tumor of $14 / 8,5 / 13 \mathrm{~cm}$. The peripheral smear was with mononuclear cells, most of them being blast cells.
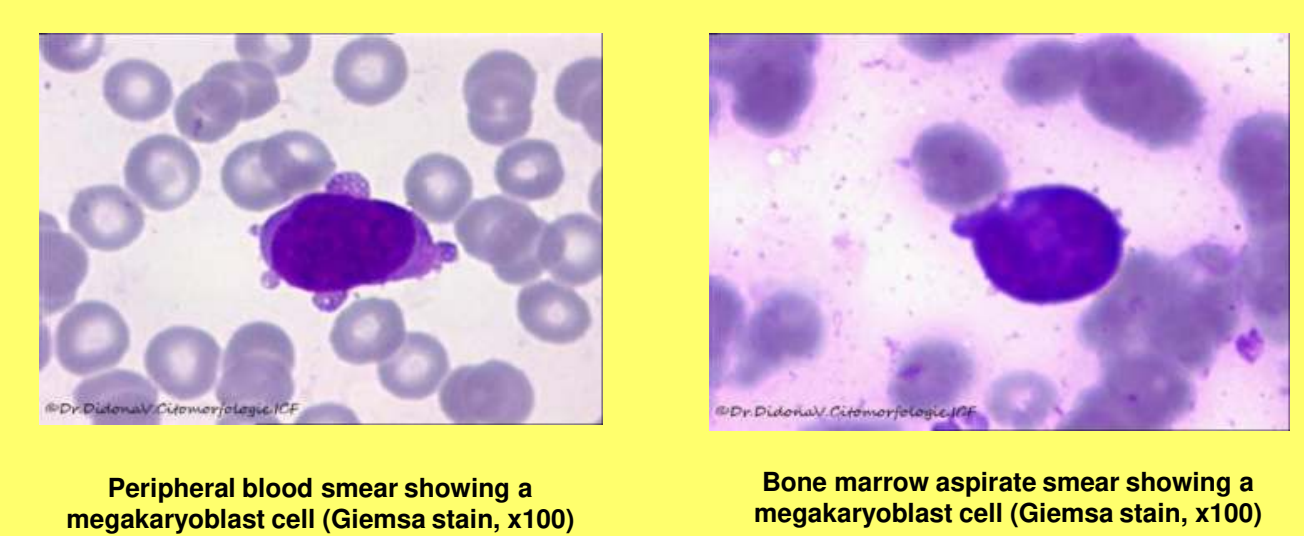

Furthermore, the first bone marrow aspiration was a dry tap and it was redone the next day showing reduced cellularity with rare megakaryocytes.

\section{FLOW CYTOMETRY}

Flow cytometry was first done on peripheral blood starting with a screening tube followed by markers for $T$ cell lymphoma and for myeloid lineage. The results of immunophenotyping ware suggestive for megakaryocyte progenitor cells and myelodysplasia and they ware emphasized by staining the bone marrow aspiration. The highlighted markers were: CD45-, cyMPO-, cyCD79-, cyCD3-, CD7+low, CD15-, CD34-, CD36+low, CD38+ ${ }^{\text {high }}, \quad$ CD56+high, CD41a+, CD42a+, CD61+, CD71+, CD117+, HLA-DR-

The molecular biology test was negative for AML (AML1-ETO-t(8;21)(q22;q22); CBFBMYH11-inv(16)(p13;q22); PML-RARA-t(15;17) (q22;q21); MLL-AF9 - $\mathrm{t}(9 ; 11)$ (p22;q23); FLT3ITD) and for ALL (E2A-PBX1-t(1;19)(q23;p13); MLL - AF4-t (4;11) (q21;q23); BCR-ABLp190 $\mathrm{t}(9 ; 22) \quad(\mathrm{q} 34 ; \mathrm{q} 11) ; \quad$ BCR-ABLp210 - $\mathrm{t}(9 ; 22)$ (q34;q11); TEL-AML1-t (12;21) (p13;q22); mSILTAL 1-del(1) (p32;p32)).
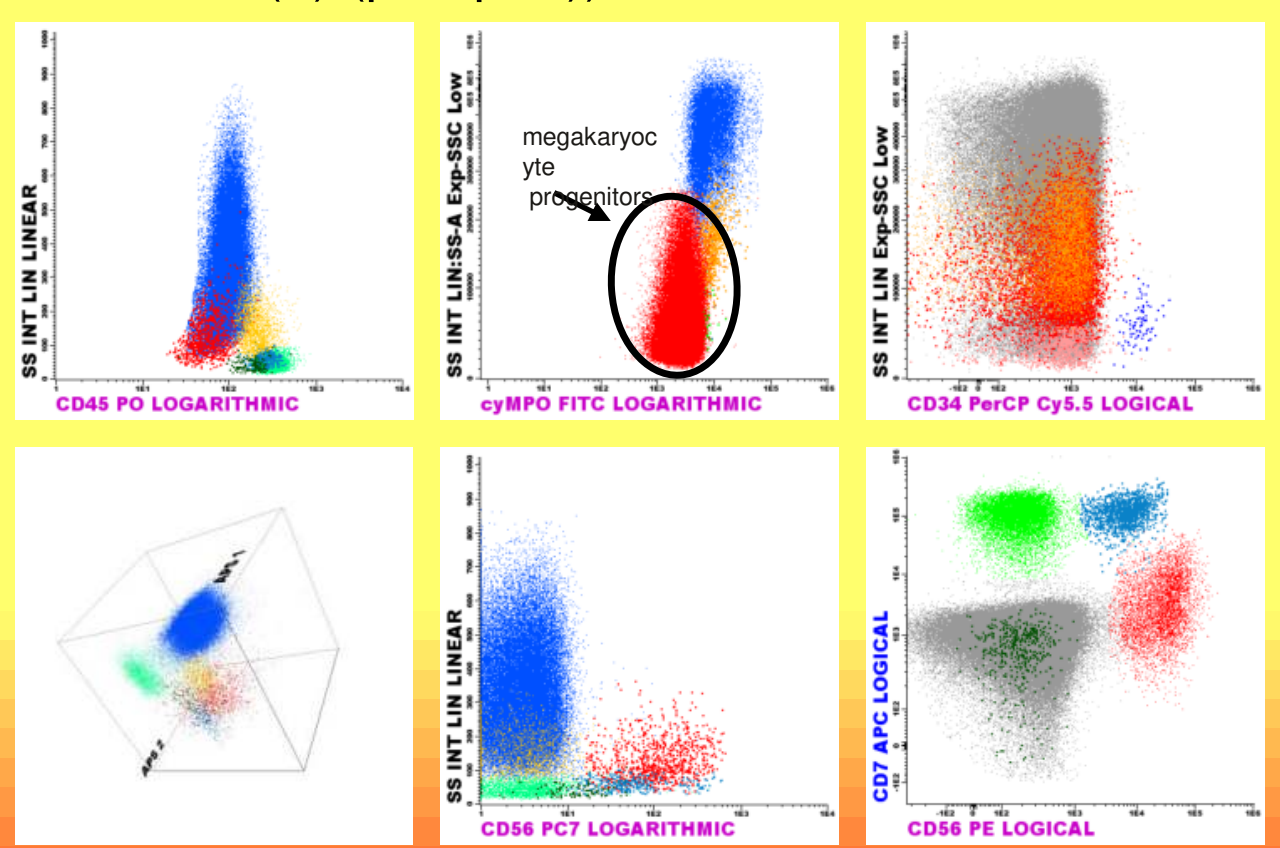
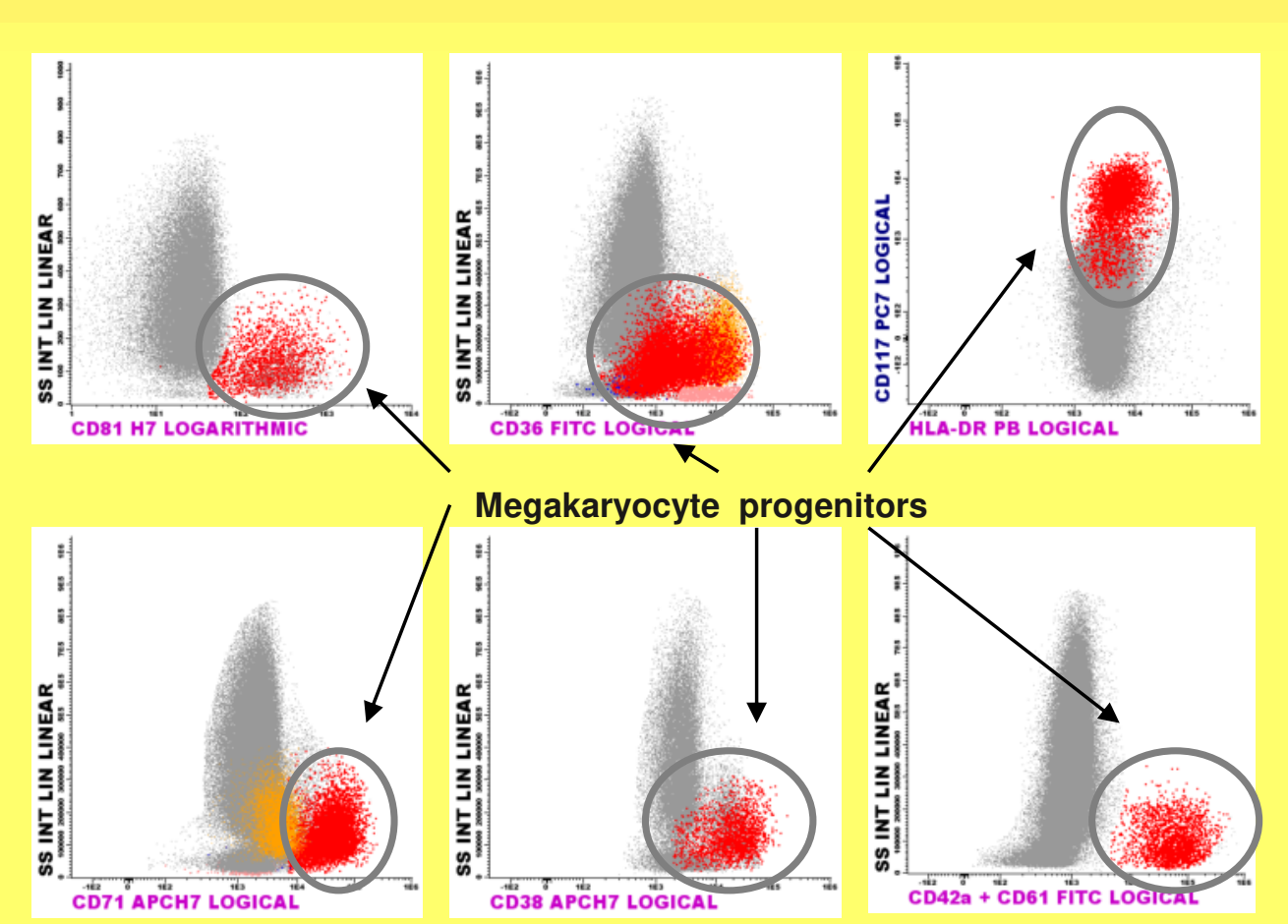

The bone marrow biopsy was packed with cells and was infiltrated by immature myeloid cells $(35-40 \%)$ that were positive for CD61 and CD56 on immunohistochemistry.
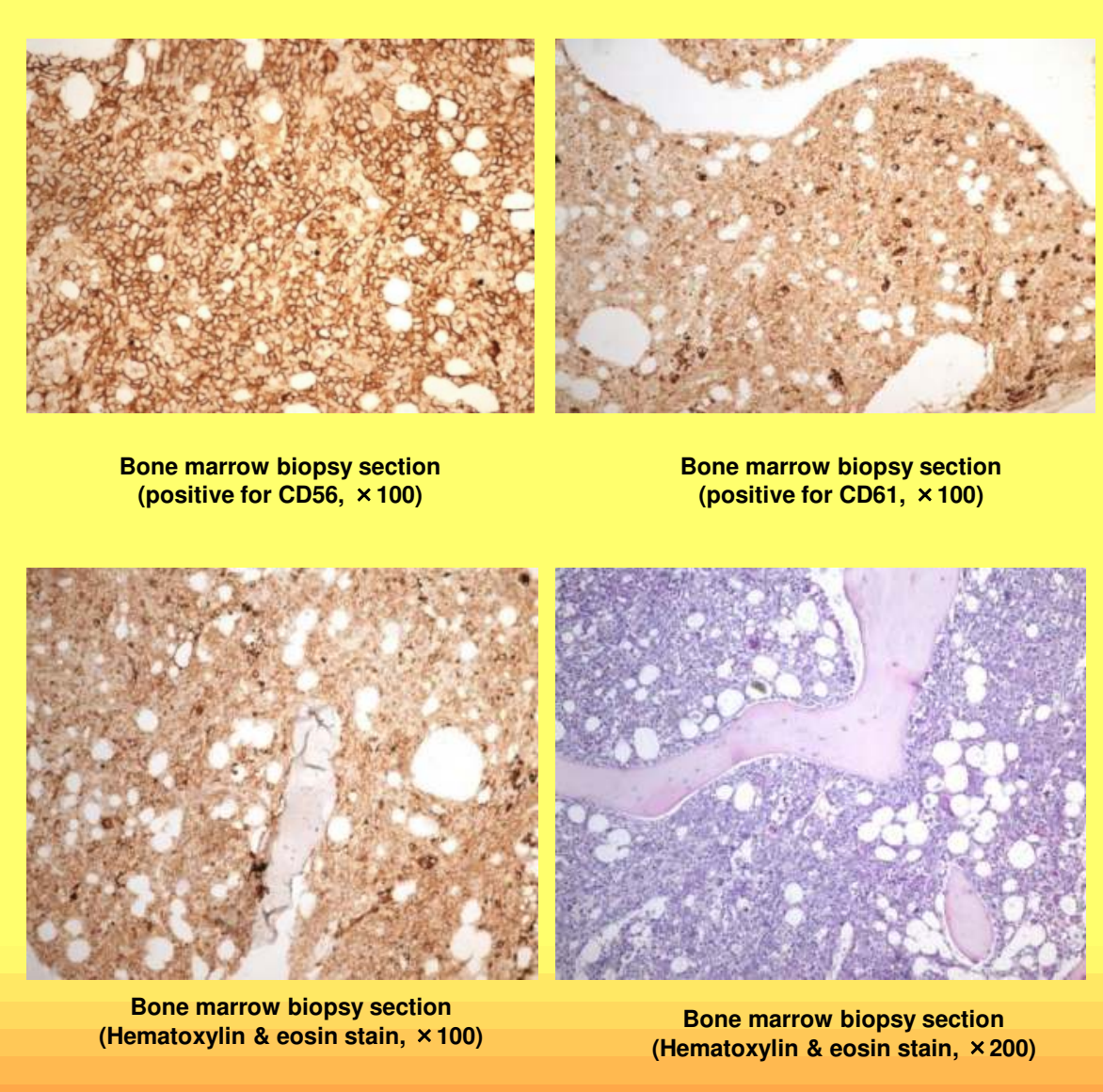

During admission to the Hematology Department, the patient's condition worsened, he developed an acute severe episode of hypotension in the context of a cardiac tamponade and a hemothorax by acute bleeding at the level of the mediastinal tumor mass. Intubation and mechanical ventilation were needed. Necessity chemotherapy was started and maintained for 3 days combining epirubicin with etoposide. The patient remained intubated during the entire aplasia period. On day +18 there were no blasts found in the peripheral blood smear.

Against all taken measure we lost the patient on day +24 due to a fungal septic sock with Candida Krusei and the autopsy confirmed the diagnosis of Acute Megakaryocytic Leukemia associated with a Mediastinal Nonseminomatous Germ Cell Tumor.

\section{CONCLUSIONS}

This case highlights the challenges faced in the diagnosis and management of this patient with acute megakaryocytic leukemias associated with mediastinal nonseminomatous germ cell tumors.

\section{REFERENCES}

Sarbajit Mukherjee et al, Ann Hematol, 2017, Sep:96(9):1435-1439. Chowdhary GS, Jhala MD J Mar Med Soc 2017:19:55-7. 